\title{
Dose-efficient strain mapping with high precision and throughput using cepstral transforms on 4D-STEM data
}

Harikrishnan K. P. ${ }^{1}$, Dasol Yoon ${ }^{2}$, Yu-Tsun Shao ${ }^{3}$, Luigi Mele ${ }^{4}$, Christoph Mitterbauer ${ }^{4}$ and David Muller ${ }^{1}$

${ }^{1}$ School of Applied and Engineering Physics, Cornell University, Ithaca, NY, USA, Ithaca, New York, United States, ${ }^{2}$ Department of Materials Science and Engineering, Cornell University, Ithaca, NY, USA, United States, ${ }^{3}$ School of Applied and Engineering Physics, Cornell University, Ithaca, NY, USA, Ithac, New York, United States, ${ }^{4}$ Materials and Structural Analysis Division, Thermo Fisher Scientific, Achtseweg Noord, Eindhoven, 5651 GG, Netherlands, Netherlands

Strain mapping of nanomaterials with high precision and spatial resolution is becoming increasingly important in the semiconductor industry, with typical industry targets of $0.01-0.1 \%$ and $1 \mathrm{~nm}$ respectively. Here, we demonstrate the potential of the exit wave power cepstral (EWPC) transform [1] as a fast and dose-efficient technique to perform strain mapping using 4D-STEM datasets. This method exploits the high dynamic range and fast readout speed of modern direct electron detectors, with the precision determined by the maximum dose, and the largest usable angle, rather than the number of pixels. The EWPC transform exploits the periodicity in the diffraction pattern rather than the exact positions of the diffracted disks and hence works well even with only a few pixels per diffracted disk leading to more compact, memory-efficient data sets. Moreover, this technique does not require any pre-processing or parameter tuning based on experimental conditions, and reduces diffraction artefacts from tilt and thickness. These factors combined with a very fast runtime (1-10 ms/pixel) makes this technique potentially well suited for high-throughput applications.

We performed strain mapping on silicon wedges and benchmarked the performance of the EWPC technique with other popular strain mapping algorithms. We find that 4D STEM data collection on the EMPAD detector [2] combined with the EWPC transform allow for fast and high precision strain mapping over large fields of view. The algorithm is also compatible with precession electron diffraction which helps to further improve precision.

Strain maps of silicon samples over large fields of view are used to show the robustness of the method to thickness and tilt artefacts. Fig. 1 shows the (a) virtual dark field image from the (11-1) reflection of a silicon wedge sample oriented along the [110] zone axis and the corresponding strain maps obtained using (b) EWPC on an EMPAD (dose $=0.2 \mathrm{pC}, 10 \mathrm{~ms} / \mathrm{pixel}$ ) and (c) industry standard NBD Strain Analysis software on a $16 \mathrm{M}$ pixel CMOS camera (dose $=0.5 \mathrm{pC}, 50 \mathrm{~ms} /$ pixel). Systematic errors from thickness fringes and defects visible in the dark field image are more suppressed in (b) compared to (c). The EMPAD's ability to record large currents at high speed and high DQE makes it possible to work with 2.5x lower dose and 5x faster acquisition compared to the low-saturation-current CMOS and MAPS detectors.

The precision achievable for strain mapping is largely determined by the dose as shown in Fig. 2(a), where the precision is plotted as a function of dose $(\mathrm{N})$ for different values of semi-convergence angle $(\alpha)$ on a $\log -\log$ scale. The dominant effect is a scaling of the precision with the Poisson noise, $1 / \sqrt{ } \mathrm{N}$. The precision improves with increasing dose, until it plateaus out when systematic errors start to dominate over the Poisson noise. The precision also improves as $\alpha$ is decreased, although this results in a drop in resolution. This trade-off is shown in Fig. 2(b) where the precision is plotted as a function of the probe size on a log- 
$\log$ scale. Given the coupling between source and beam current, to achieve a $0.1 \%$ precision at $1 \mathrm{~nm}$ resolution, one would be better off using $\alpha=2 \mathrm{mrad}$ rather than 1.6 or $3 \mathrm{mrad}$.

Using the EWPC method, a measured precision of $0.09 \%$ and a resolution of $1 \mathrm{~nm}$ is achieved with a dose of $1 \mathrm{pC}$ with $\alpha=2 \mathrm{mrad}$, recorded with a dwell time of $10 \mathrm{~ms}$. Replacing the Schottky with CFEG source will reduce the dwell time need by $\sim 3-5 x$. The precision values obtained with the EWPC method are comparable to or slightly better than the precision values reported in literature for large-pixel-format detectors using other algorithms [3-6]. As processing times also scale with the number of pixels, the ability to use small data sets, considerably speeds up the computing time. Different algorithms and their fine tuning can result in small improvements in precision, but for a large order of magnitude improvement, the only avenue is to use a higher dose. This requires collecting the largest dose on the sample in the shortest time, i.e. the largest saturation current per pixel, which is $\sim 2 \mathrm{pA} /$ pixel for the EMPAD1 and $\sim 180$ $\mathrm{pA} /$ pixel for our new prototype.
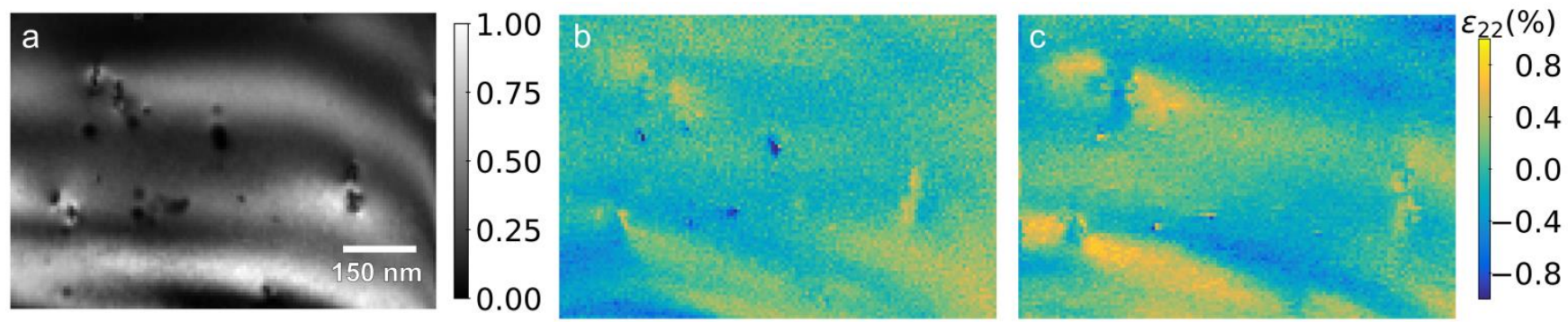

Figure 1. Robustness of EWPC strain mapping to thickness artefacts in comparison to industry grade software. (a) Virtual dark field image from the (11-1) reflection of a silicon sample oriented along the [110] zone axis. Thickness fringes and defects over the field of view can be clearly seen. Strain maps over the same region obtained using the (b) EWPC method (dose $=0.2 \mathrm{pC}, 10 \mathrm{~ms} / \mathrm{pixel}$ ) with data collected on the 128 x 128 pixel EMPAD detector and (c) Commercial Strain Analysis software with data collected on a $16 \mathrm{M}$ pixel CMOS camera (dose $=0.5 \mathrm{pC}, 50 \mathrm{~ms} / \mathrm{pixel})$. A semi-convergence angle of $0.6 \mathrm{mrad}$ is used in both cases.
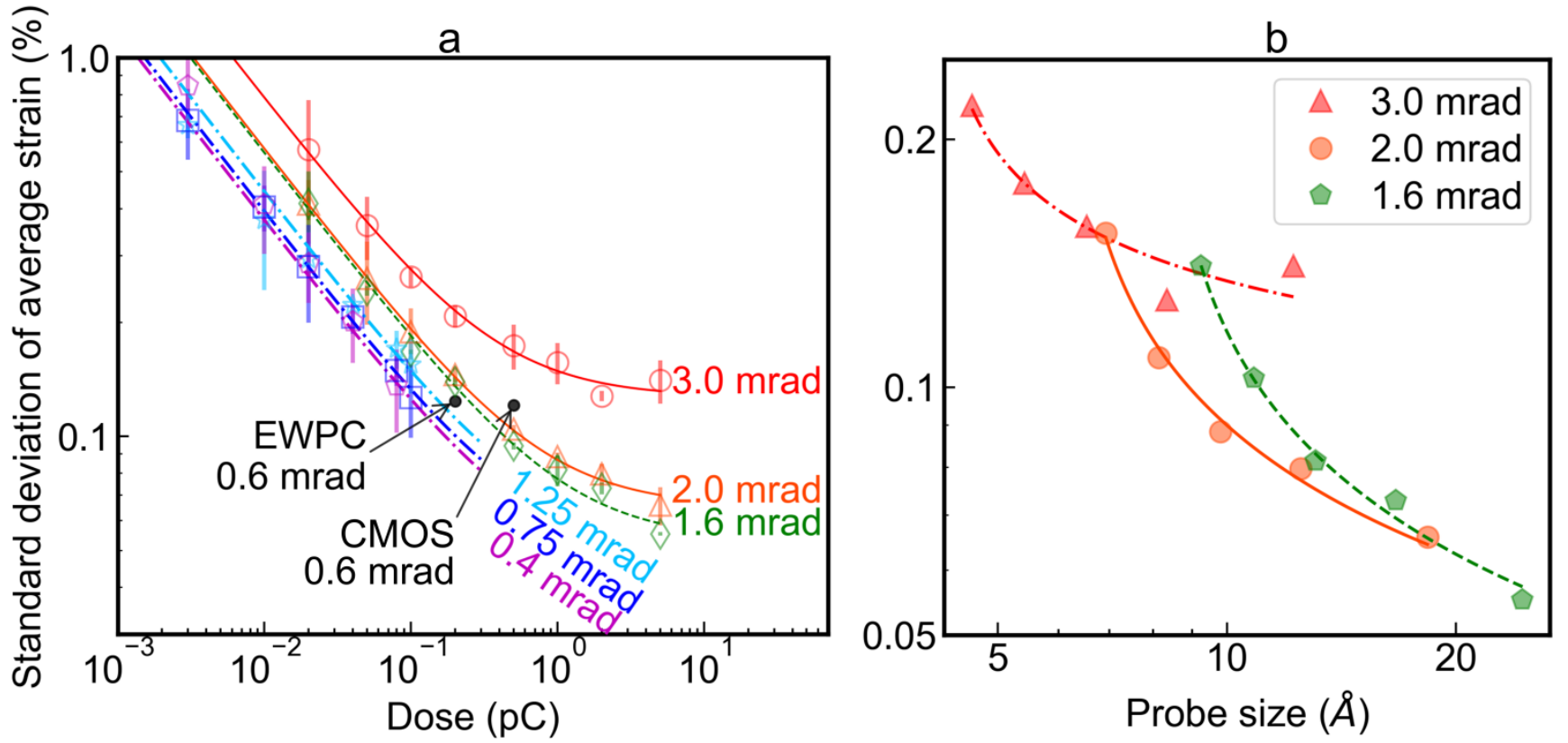
Figure 2. Measured local precision for strain mapping with different experimental conditions. The mean of the precision of the diagonal components of the strain matrix is used as the data point with the standard deviation as the error bar. (a) Precision as a function of dose $(\mathrm{N})$ on a log-log scale plotted for different values of probe semi-convergence angle $(\alpha)$. The data points are fit with a function of $1 / \sqrt{ } \mathrm{N}$ from the Poisson noise added in quadrature with a constant from other noise sources. The precisions measured from same region of the data sets in Figure 1 for both the EWPC/EMPAD and CMOS data are also marked (different sample thickness to colored data series). (b) Precision as a function of the probe size on a loglog scale, showing the trade-off between higher precision and better resolution.

\section{References}

[1] E. Padgett, et al., Ultramicroscopy 214, 112994 (2020).

[2] M. W. Tate, et al., Microsc. Microanal. 22, 237 (2016).

[3] R. Yuan, et al., Ultramicroscopy 207, 112837 (2019).

[4] K. Müller, et al., Appl. Phys. Lett. 101, 212110 (2012).

[5] V. B. Ozdol, et al., Appl. Phys. Lett. 106, 253107.1 (2015).

[6] D. Cooper, et al., Nano Lett. 15, 5289 (2015). 